“(C) 2014 IEEE. Personal use of this material is permitted. Permission from IEEE must be obtained for all other uses, in any current or future media, including reprinting/republishing this material for advertising or promotional purposes, creating new collective works, for resale or redistribution to servers or lists, or reuse of any copyrighted component of this work in other works." 


\title{
Compact Hyper-Band Printed Slot Antenna with Stable Radiation Properties
}

\author{
Ming-Chun Tang, Member, IEEE, Richard W. Ziolkowski, Fellow, IEEE, and Shaoqiu Xiao, Member, IEEE
}

\begin{abstract}
A compact hyper-band (> 10:1 impedance bandwidth) printed antenna design is investigated numerically and experimentally. It is based on an elliptical-slot antenna augmented with a parasitic oval patch and driven with a specially engineered microstrip-line-fed elliptical tuning fork element. The parasitic and driven elements are adjusted along with the elliptical slot to create additional resonance modes; adjust the coupling strengths among all of the design components; facilitate the overlap of adjacent resonance modes; and fine tune the input impedance. The total size of the final optimized antenna is only $30 \times 40 \mathrm{~mm}^{2}$. It exhibits a -10-dB impedance bandwidth from 2.26 to $22.18 \mathrm{GHz}$. Desirable radiation performance characteristics, including relatively stable and omni-directional radiation patterns, are obtained over this range. A prototype was fabricated and tested. The experimental results confirm the predicted input impedance bandwidth and radiation characteristics. While the hyper-band performance could be used for high fidelity short pulse applications, the antenna could also be used for multi-band operations from 3.1-10.6 GHz since it covers that entire ultra-wideband (UWB) spectral range.
\end{abstract}

Index Terms - Compact antennas, hyper-band, parasitic elements, printed slot antennas, ultra-wideband antenna

\section{INTRODUCTION}

$\mathrm{P}$ RINTED slot antennas have received serious attention for use in numerous wireless communication systems due to their many attractive practical characteristics, including low profile, light weight, cheap manufacturing, planar geometry, ease of integration with other microwave devices, and so on [1, 2]. In recent years, possessing favorable wideband impedance bandwidth characteristics, various wide-slot antennas having different slot shapes have been reported. These include rectangle [3], ellipse [4], arc [5], heptagon [6], binomial curve [7], spiral [8], rhombus [9], inverted cone [10], L-shape [11], triangle [12, 13], circle [14, 15], fractal [16-18], and rotated square [19-21] designs. Concomitantly, the directly driven elements in most of these antennas have specially engineered

Manuscript received 28 July, 2013; revised 18 December, 2013; revised 09 February, 2014; and revised 21 March 2014.

This work was supported in part by the National Natural Science Foundation of China contract number 60971029 and in part by NSF contract number ECCS-1126572.

M.-C. Tang is with the College of Communication Engineering, Chongqing University, Chongqing, 400044, China, also with the Institute of Applied Physics, University of Electronic Science and Technology of China, Chengdu, 610054, China (E-mail: tangmingchunuestc@ 126.com);

R. W. Ziolkowski is with the Department of Electrical and Computer Engineering, University of Arizona, Tucson, AZ 85721 USA (E-mail: ziolkowski@ece.arizona.edu);

S. Xiao is with the Institute of Applied Physics, University of Electronic Science and Technology of China, Chengdu, 610054, China. terminations of their feed lines to achieve suitable mutual coupling with the slots for optimum impedance matching levels over as wide a frequency range as possible. These terminations include fork-like- [4], square-patch- [5], leaf- [10], W- [11], triangle- [12], and circle- [15] shaped stubs.

Theoretically, all of these printed wide-slot designs [3-21] originate from the basic rectangle-shaped slot antenna [1]. They all possess an inherent abundance of resonance modes [2], the mode density increasing as the driving wavelength decreases. However, before effective tuning, their resonance frequency bands are not overlapping nor input impedance matched, for example, to a $50 \Omega$ source. This undesirable behavior is illustrated in Fig. 1(a).

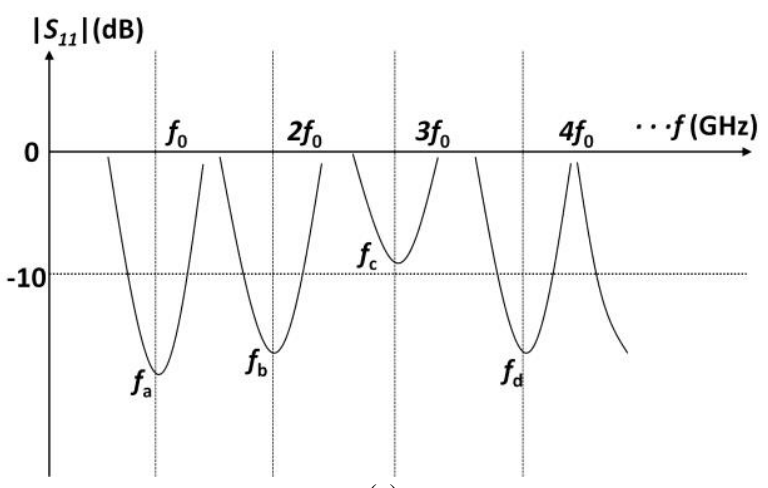

(a)

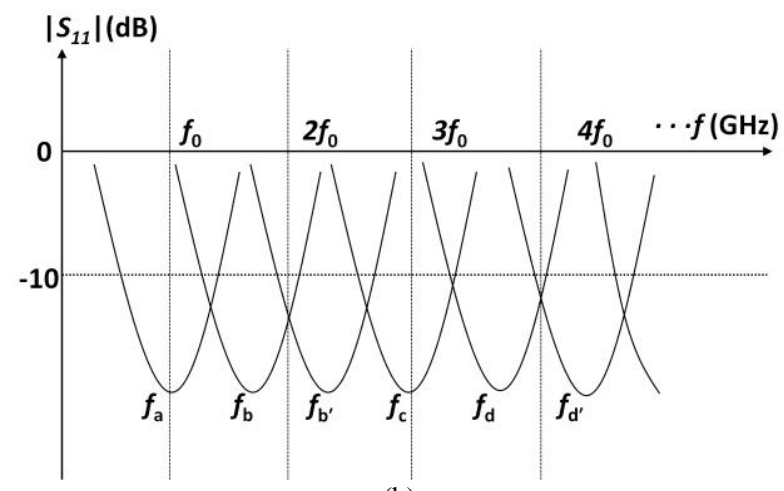

(b)

Fig. 1 Schematic of the impedance characteristics $\left(\left|S_{11}\right|\right)$ as a function of frequency. (a) Common printed wide-slot antenna before tuning; (b) proposed printed wide-slot antenna after refined tuning.

Usually, by tuning the stub and slot dimensions, the overlap and impedance matching characteristics of these resonances can be adjusted effectively to realize the desired large instantaneous impedance bandwidth. Moreover, as was reported in [4, 10, 15], if the ground is made large enough, these stub modifications can 
be exploited to merge several nearby frequency bands associated with the lower-order modes to achieve even wider bandwidths (e.g., $-10 \mathrm{~dB}\left|\mathrm{~S}_{11}\right|$ level bandwidths of about $112.6 \%$ in [4], higher than $172.7 \%$ in [10], and up to $143.2 \%$ in [15]).

However, when the ground size is comparable to the slot size, the bandwidths are confined to a relatively narrower range (only $90.6 \%$ in [18]). This constraint occurs because of the significant negative effect of the ground edge on the mutual coupling between the feed line and slot. Thus it is necessary in a compact printed slot antenna design to embed parasitic elements to adjust the coupling levels in a wide frequency range instead of only resorting to tuning the stub dimensions. A parasitic square patch was embedded in the center of the slot to enlarge the resonant frequency interval between the first and second modes in [20]. This approach successfully enhanced the impedance bandwidth up to $82.3 \%$. In another manner, a pair of parasitic strips was embedded along the feed line to excite more resonant modes in [9]. An impedance bandwidth of $108.7 \%$ was obtained. As a combination of these approaches, a much larger impedance bandwidth (136.4\%) was realized in [21] by incorporating both a parasitic square patch and parasitic feed-line strips into a slot antenna design, while maintaining its compact size. Nonetheless, a printed slot antenna that not only has a very large impedance bandwidth, but also has a more compact size than the one reported in [21], would be desirable for a number of wireless applications.

In this paper, benefitting from the inherent abundance of resonance modes associated with an elliptical slot antenna, a parasitic oval patch is embedded in the slot to create additional resonance modes and to facilitate the overlap of various combinations of both sets of modes. Moreover, to provide additional tuning capabilities, the micro-strip line that feeds the slot is terminated in an elliptical-shaped tuning fork stub engineered to merge the resonance modes and to adjust their input impedance values to achieve nearly complete matching to a $50 \Omega$ source over a hyper-band $\left(2 *\left(f_{h}-f_{l}\right) /\left(f_{h}+f_{l}\right)>163.4 \%\right.$ half-power fractional bandwidth with a >10:1 bandwidth ratio, $f_{h} / f_{l}$, defined by the upper, $f_{h}$, and lower, $f_{l}$, half-power frequency points, a terminology borrowed from high power microwave (HPM) applications [22]), while maintaining a compact size. The goal of the resulting design for its $\left|S_{11}\right|$ values is illustrated in Fig. 1(b). The resulting hyper-band design is suitable for short-pulse HPM applications. Moreover, because its hyper-band covers the standard UWB band, 3.1-10.6 GHz, it is demonstrated that the radiation characteristics of this antenna are also very suitable simply for multi-band operation in that range.

\section{ANTENNA DESIGN}

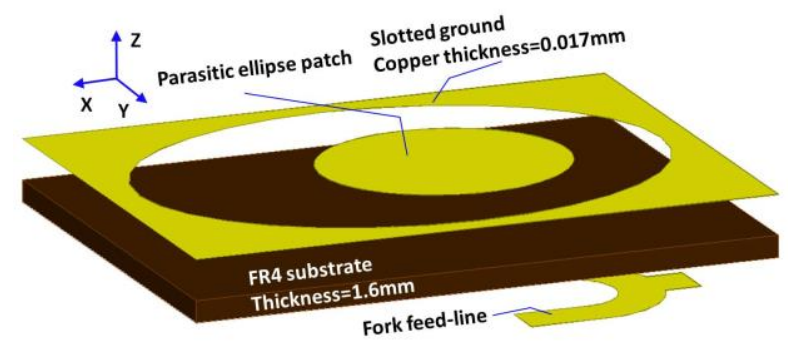

(a)

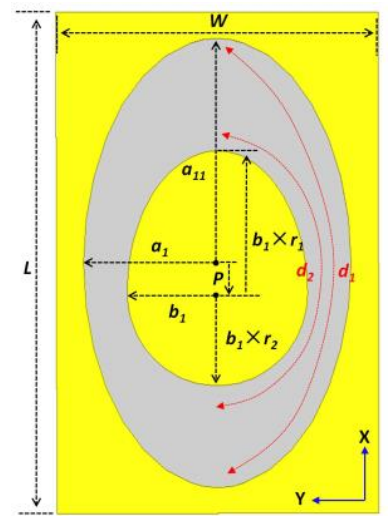

(b)

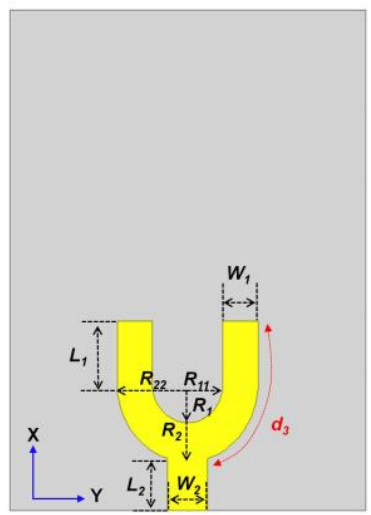

(c)
Fig. 2 Configuration of the proposed printed hyper-band antenna. (a) 3-D, (b) top and (c) back views. Design parameters (all dimensions in $\mathrm{mm}$ ): $L=40$, $W=30, L_{1}=5, L_{2}=2.347, W_{1}=2.9545, W_{2}=3.35, R_{1}=3.14, R_{2}=6.25, R_{11}=2.983$, $R_{22}=5.9375, p=2.4, a_{1}=12.4584, a_{11}=17.9, b_{1}=8.4, r_{l}=1.35, r_{2}=0.88$, $d_{1}=48.071, d_{2}=28.005$ and $d_{3}=12.873$.

The geometry of the proposed antenna is illustrated in Fig. 2. This design is intended to be constructed using printed circuit board (PCB) technology. It presumes an FR4 substrate with the dielectric constant $\varepsilon_{r}=4.4$ and loss tangent $\tan \delta=0.02$. Its overall size is only $L \times W \times H=40 \mathrm{~mm} \times 30 \mathrm{~mm} \times 1.6 \mathrm{~mm}$. It has a simple configuration. An elliptical shaped slot is centered on the top side of an FR4 board. An oval shaped (two conjoined half ellipses) parasitic patch located in the slot with its center displaced from the slot center by $p=2.4 \mathrm{~mm}$. On the bottom side, a microstrip feed line designed to have a $50 \Omega$ characteristic impedance (width $W_{2}=3.35 \mathrm{~mm}$ ) is terminated in a fork-shaped tuning stub. The choice of an elliptical shape for this stub is a natural extension from the slot shape choice; it increases the tuning degrees of freedom for impedance matching [4, 23]. Note that, without any parasitic element loading of the slot, the design would be incapable of achieving the desired hyper-band performance. In our simulation studies, no matter how the tuning stub and/or slot dimensions were modified, impedance matching could not be accommodated over such a wide frequency band. Typically, as is demonstrated in Fig. 3, when changing the tuning fork length $\left(L_{1}\right)$, the impedance matching in the lower bands could be improved, but would degrade in the higher bands as a trade-off. The refinements of the design were obtained numerically with both the ANSYS/ANSOFT high frequency structure simulator (HFSS) and CST's Microwave Studio (MWS). These design studies are discussed in Section III and in the Appendix. 


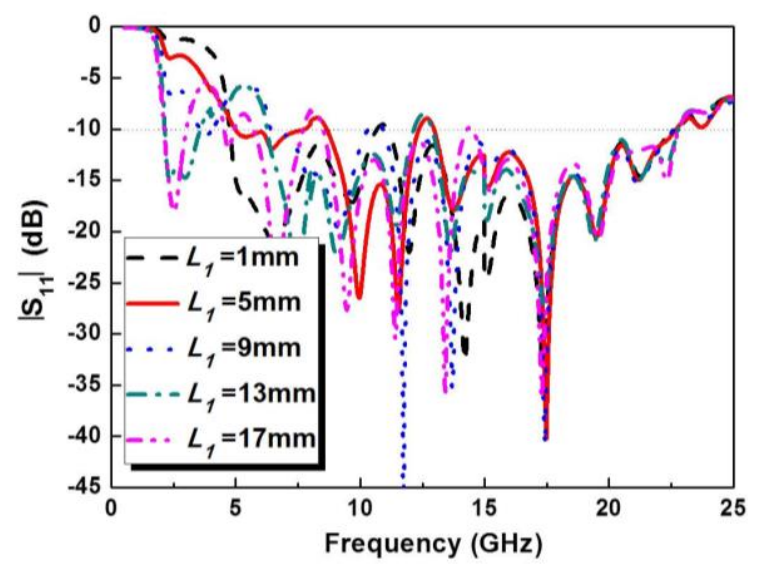

Fig. 3 Effect of the fork length $\left(L_{I}\right)$ on the input impedance characteristics of the slot antenna in Fig. 2 when the parasitic oval disc is absent.

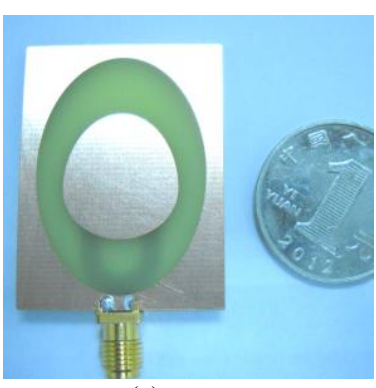

(a)

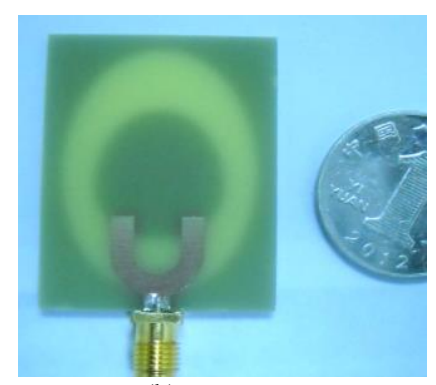

(b)
Fig. 4 Fabricated prototype of the antenna. (a) Front view, and (b) back view.

The antenna shown in Fig. 2 was fabricated and its performance characteristics were obtained experimentally. The prototype is shown in Fig. 4. First, the reflection coefficient of the antenna was measured using Agilent E8361A PNA Vector Network Analyzer (VNA) with the measurement scale $0.01 \mathrm{GHz}-67 \mathrm{GHz}$. The measured results together with the corresponding simulation values are plotted in Fig. 5. As anticipated from the HFSS (CST) simulation results, the antenna could provide a $>10: 1$ impedance matching bandwidth with $\left|\mathrm{S}_{11}\right|<-10 \mathrm{~dB}$ from 2.17 (2.16) to $23.01 \mathrm{GHz}(22.72 \mathrm{GHz})$. The experimental results demonstrate that the antenna provided a -10dB-level impedance bandwidth from 2.26 to $22.18 \mathrm{GHz}$ ( $163.01 \%$ fractional bandwidth, $~ 10: 1$ bandwidth ratio). On the whole, the measurement and simulations agree very well with each other and provide the desired hyper-band performance (note that the half-power bandwidth values are even larger than the reported $-10 \mathrm{~dB}$ bandwidths). Furthermore, in comparison with the combination reported in [21], the proposed antenna provides not only a much wider impedance bandwidth, but also has a simpler configuration (i.e., it uses fewer parasitic elements) and a more compact size ( $12.3 \%$ size reduction). Note that the measured frequencies are shifted about $0.1 \mathrm{GHz}$ higher than the simulation results due to the presence of a long coaxial cable used in the measurements. We believe the slight decrease in the measured bandwidth at the upper bound is due to minor fabrication issues.

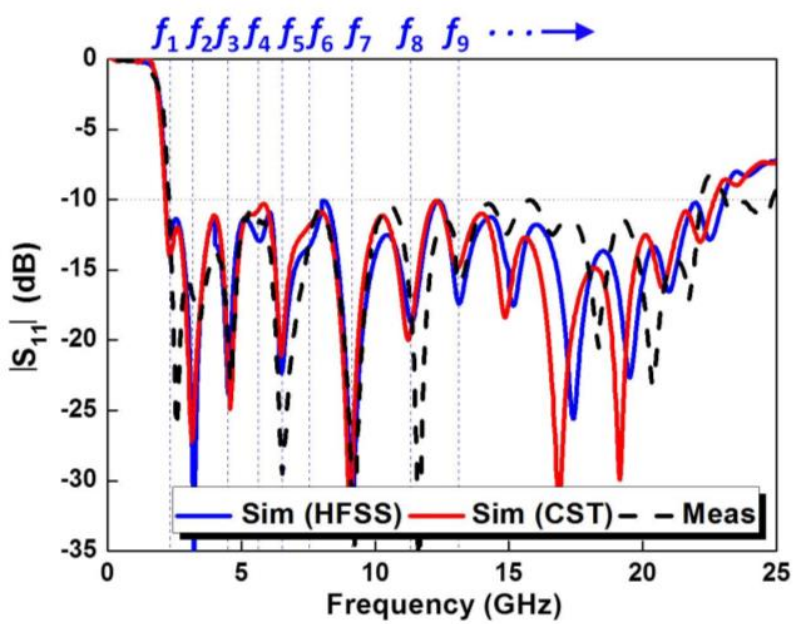

Fig. 5 Measured and simulated input impedance characteristics $\left(\left|\mathrm{S}_{11}\right|\right.$ in $\left.\mathrm{dB}\right)$ of the slot antenna shown in Figs. 4 and 2.

The far-field radiation patterns were measured in an anechoic chamber, which is mainly based on the Agilent EB362C PNA VNA and SATIMO passive measurement system (with the measurement range 0.8-18.0 GHz). The measured results together with the corresponding simulation values are shown in Figs. 6 and 7. As Fig. 6 demonstrates, the $E$-plane pattern is monopole-like. The number of lobes increases with an increase of the frequency, which indicates that the antenna operates in higher-order modes and gets more directional as the frequency increases [4, 24]. The slight asymmetry found in the experimentally-obtained $E$-plane patterns, especially in the lower bands, is mainly attributed to the coaxial cable contribution on the radiation characteristics. This arises because of the compact size of the antenna, which causes the slot to be quite close to the coaxial cable (shown in the Fig. 4). Fig. 7 also includes the angles at which the simulated peak values occur relative to the broadside direction in the $E$-plane in the upper hemisphere of the antenna (+ z-axis). The HFSS and CST results are in very good agreement. The variations of these peak directions in the $E$-plane are associated with the additional modes that are introduced as the frequency increases. One finds smooth transitions in the patterns and the peak value directions as each of these additional modes appears. The $H$-plane pattern is nearly omni-directional in the lower frequency range (2.26-12.0 GHz). However, it becomes more asymmetrical and less smooth above $12 \mathrm{GHz}$. This degradation of its omni-directional character is attributed to the combination of several properties. One, with the increase in frequency, the order of the dominant slot resonance mode becomes higher. Second, the presence of the parasitic patch leads to more resonant modes, which combine with the slot modes to yield complicated modal distributions. Third, the patch itself also resonates at the higher frequencies. Accordingly, it contributes to the radiation performance in the higher frequency bands. Fourth, the feed line becomes resonant at the higher frequencies and thus also impacts the radiation performance for those frequencies. 


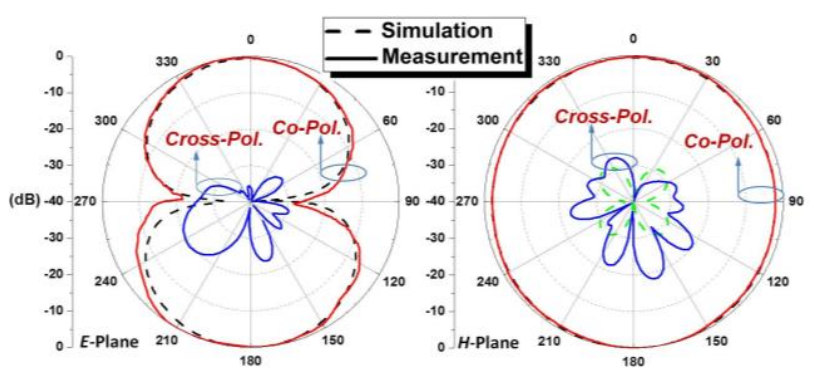

(a)

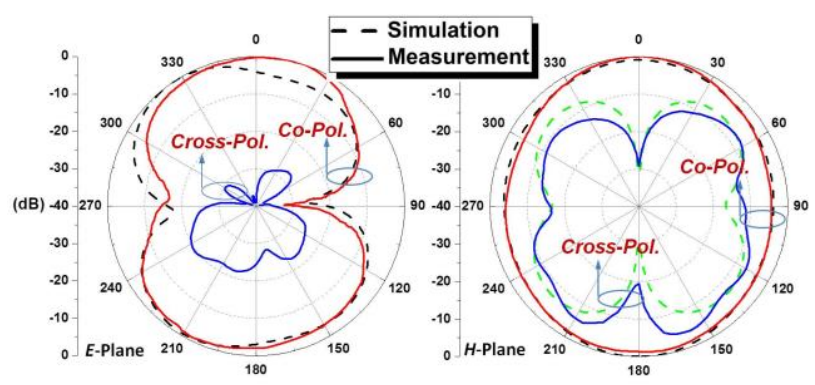

(b)

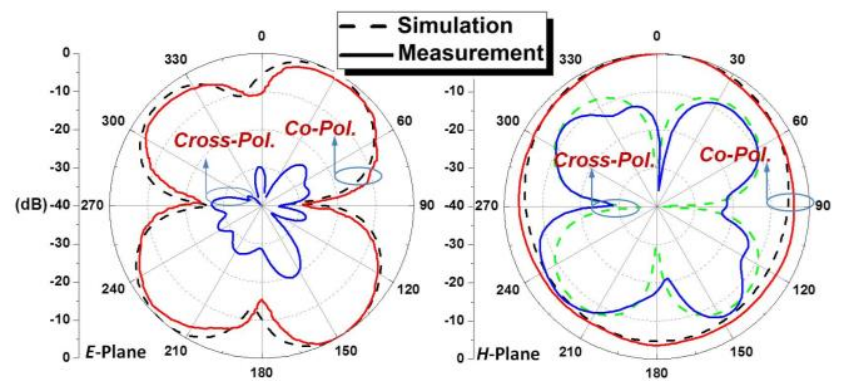

(c)

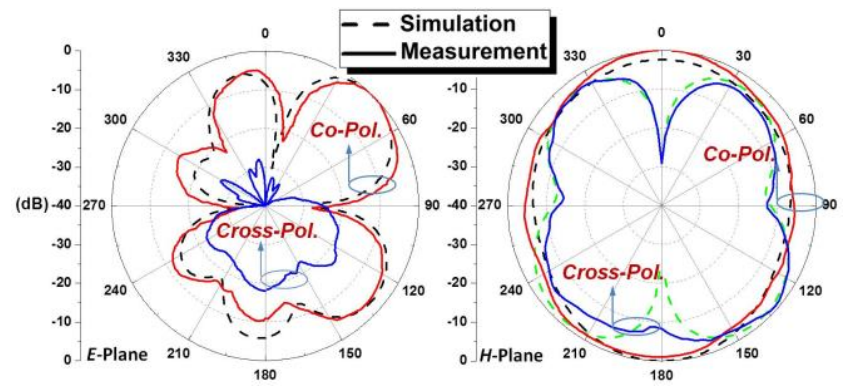

(d)

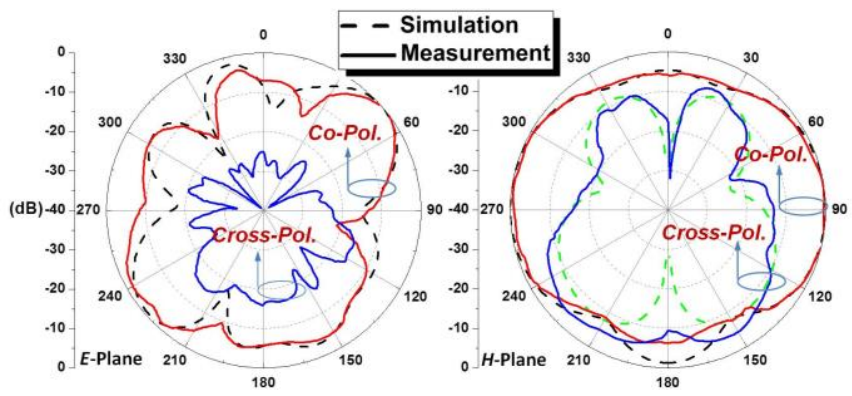

(e)

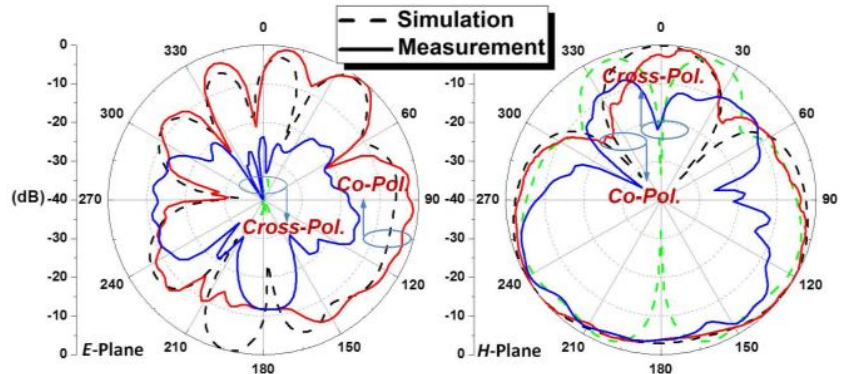

(f)

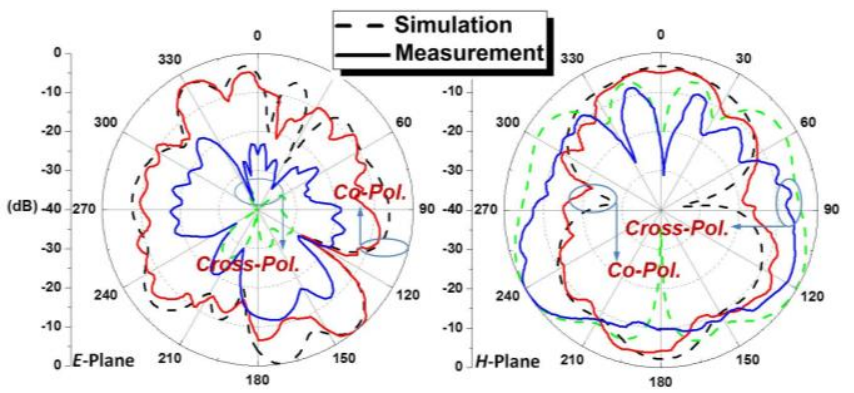

(g)

Fig. 6 HFSS simulated and measured normalized radiation patterns. (a) $3.0 \mathrm{GHz}$ (b) $4.5 \mathrm{GHz}$, (c) $6.0 \mathrm{GHz}$, (d) $9.0 \mathrm{GHz}$, (e) $12.0 \mathrm{GHz}$, (f) $15.0 \mathrm{GHz}$, and $(\mathrm{g}) 18.0 \mathrm{GHz}$.

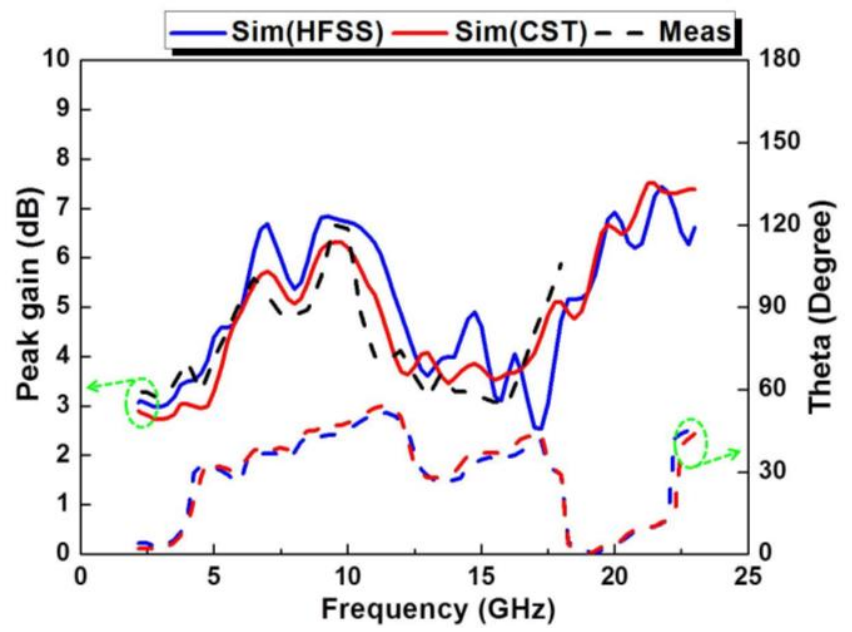

Fig. 7 Measured and simulated peak gain values and the angle $\theta$ away from the $+\mathrm{z}$ axis at which the simulated peak value occurs in the forward hemisphere of the $E$-plane versus frequency $(2.16 \mathrm{GHz}-23 \mathrm{GHz})$.

TABLE I. HFSS SIMULATED RADIATION CHARACTERISTICS OF THE HYPER-BAND UWB ANTENNA WITH (SHADED) AND WITHOUT THE PARASITIC OVAL PATCH.

\begin{tabular}{|c|c|c|c|c|}
\hline \multicolumn{2}{|c|}{ Typical frequency } & $\begin{array}{l}\left|\mathrm{S}_{11}\right| \\
(\mathrm{dB})\end{array}$ & $\begin{array}{l}\mathrm{OE} \\
(\%)\end{array}$ & $\begin{array}{l}\text { Peak realized } \\
\text { gain }(\mathrm{dB})\end{array}$ \\
\hline \multirow{6}{*}{ WiMAX } & \multirow{2}{*}{$2.5 \mathrm{GHz}$} & -11.43 & 92.52 & 2.70 \\
\hline & & -2.96 & 45.51 & -0.09 \\
\hline & \multirow{2}{*}{$3.5 \mathrm{GHz}$} & -16.70 & 95.75 & 3.02 \\
\hline & & -4.23 & 59.13 & 1.51 \\
\hline & \multirow{2}{*}{$5.5 \mathrm{GHz}$} & -12.47 & 88.41 & 4.52 \\
\hline & & -10.74 & 86.36 & 4.91 \\
\hline \multirow{6}{*}{ WLAN } & \multirow{2}{*}{$2.4 \mathrm{GHz}$} & -12.03 & 93.73 & 2.75 \\
\hline & & -3.07 & 46.84 & -0.01 \\
\hline & \multirow[b]{2}{*}{$5.2 \mathrm{GHz}$} & -11.40 & 87.55 & 4.50 \\
\hline & & -10.62 & 87.41 & 5.02 \\
\hline & \multirow{2}{*}{$5.8 \mathrm{GHz}$} & -12.61 & 86.95 & 4.18 \\
\hline & & -10.45 & 85.09 & 5.07 \\
\hline
\end{tabular}

We note that while our hyper-band UWB antenna 
configuration is related to the slot-ring antennas reported in [25-27], the designs of the parasitic patch and feed-line stub are quite different. The impact of the elliptical tuning fork feed-line stub was discussed previously in $[4,23]$. On the other hand, in order to emphasize the impact of the parasitic oval patch in our design, the performance characteristics of the antenna with and without this parasitic patch are compared in Table I at the center frequencies of the WiMAX and WLAN bands. It is shown that the parasitic oval patch promotes a much wider impedance bandwidth and improves the overall efficiency (OE) significantly in this lower portion of its operational band. It is also shown that there are frequencies at which the antenna has slightly higher peak realized gain values without the parasitic oval patch than with it. This effect indicates that the antenna without the parasitic patch is more directional at those frequencies [24].

In addition, Fig. 6 also shows that the cross-polarization in the higher frequency bands (above $4.5 \mathrm{GHz}$ ) is relatively high. As with other UWB antennas, e.g., [9, 21], this cross-polarization behavior results in a slight reduction of the maximum gain values. Moreover, it is found that the cross-polarization level of our antenna is lower over this frequency range as compared to the UWB antenna reported in [21]. The measured maximum gain values together with their simulated values are presented in Fig. 7. It is observed that the measured maximum gain fluctuates within the range from $3.14 \mathrm{~dB}$ to $6.59 \mathrm{~dB}$ in the range from 2.26 to $12.0 \mathrm{GHz}$. Since this frequency range covers the 2.4/5.2/5.8 $\mathrm{GHz}$ WLAN bands, $2.5 / 3.5 / 5.5 \mathrm{GHz}$ WiMAX bands, and 3.1-10.6 GHz UWB band, the proposed antenna would be a good candidate in any or all of these wireless communication applications bands. When operating in the higher frequency bands, the peak gain variation is similar to those presented previously, e.g., [4, 14]. Also, the HFSS and CST simulated radiation efficiencies over the entire operation frequency range are higher than $75 \%$, which are consistent with the HFSS and CST predicted (and measured) realized gain values. The high radiation efficiency values indicate that a lot more power is radiated than is retained in the antenna, which in turn means that it will run cooler than many other alternative designs and would make it a good candidate for long term operations with minimal performance degradations.

Note that there are minor differences between the CST and HFSS results shown in Figs. 5 and 7. These variations can be ascribed to the following two reasons. First, the simulators are different, i.e., the CST results were obtained in the time domain with a mesh appropriate for its finite integration technique (FIT) solver and the HFSS results were obtained in the frequency domain with a mesh appropriate for its finite element method (FEM) solver. Second, the values themselves were obtained differently across the entire hyperband. The HFSS simulated results were generated by dividing the entire band into several one-octave sub-bands: $0.5-1 \mathrm{GHz}, 1-2 \mathrm{GHz}, 2-4 \mathrm{GHz}, 4-8 \mathrm{GHz}$, $8-15 \mathrm{GHz}$, and $15-25 \mathrm{GHz}$, to achieve more accurate simulation results than if only one frequency range was used to cover the entire hyperband. Consequently, different radiation box sizes, which corresponded to those sub-bands, were employed. Since each sub-band contains many frequencies, it becomes inevitable that the simulation box sizes within and across sub-bands will not be the desired quarter-wavelength size for every frequency. In contrast, the CST results required only one very large problem space and very long run times. In particular, the reported results were generated by using 49.3 million hexahedral mesh cells at a cost of $13 \mathrm{~h} 41 \mathrm{~m}$ to finish on a computer with an Intel(R) Core(TM) i7-4770 CPU, 16GB of RAM, and a 64-bit operating system. Yet smaller cell sizes and longer simulation times would have provided yet more accurate simulation results. The values reported were obtained with the maximum available computer resources. Despite the small variations in the predicted performance characteristics found in Figs. 5 and 7, both simulators predicted values in very reasonable agreement with each other and the measured values.

\section{DISCUSSION}

As is well known, the bandwidth of many common resonant antennas is dictated by its fundamental mode [2]. In wideband antenna design technology, a common and effective way to achieve ultra-wideband operation is to make a certain number adjacent modes overlap with each other. This method has been emphasized in several types of UWB antenna designs [4, 28, and 29]. The simulated surface currents for several resonance modes of the fabricated antenna are given in Fig. 8. As is clearly illustrated, with an increase of the operating frequency, the resonance mode number increases. Nonetheless, from Fig. 5, the overlap of the resonance modes causes these current distributions to arise from the combination of several modes. Different from the study in [4], for example, which emphasized only four modes, the fabricated antenna utilizes a large set of resonance modes to achieve its hyper-band performance.

More specifically, by investigating the current distributions, it can be easily verified that, the antenna operates in the fundamental mode at $f_{l}$ due to the slot resonance. This resonance frequency is determined by the slot length $d_{l}$. Subsequently, the frequencies of the higher-order modes are determined by $d_{l}$ and should be $\mathrm{n} \times f_{l}$ (where $\mathrm{n}=2,3,4 \ldots$ ). They are in a sequence corresponding to the slot's second, third, fourth, etc., resonance modes. In a similar manner, the outer edges of the ground along the $x$-axis have a resonance at $f_{2}$. This frequency is determined mainly by the ground length $L$. It is noted that the parasitic oval patch length is $d_{2} \approx d_{1} / 2$ along that axis. Consequently, the resonance frequency of its fundamental mode is about $2 \times f_{1}=$ $4.56 \mathrm{GHz}$, which is quite close to the $2^{\text {nd }}$-harmonic mode frequency of the slot. Then due to the mutual coupling between the slot edge and the parasitic patch, the center frequencies of the two modes: the fundamental mode of the parasitic patch and the $2^{\text {nd }}$-harmonic mode of slot, brings these two modes closer together giving a single broad resonance at $4.56 \mathrm{GHz}$. At $f_{3}$, the current is linearly distributed on the parasitic patch in the normal $\mathrm{TM}_{10}$ mode. Moreover, this mode is much stronger than the one at $f_{4}$. Therefore, the antenna should be operating in the fundamental mode of the parasitic patch at $f_{3}$. At $f_{4}$, one finds that there are strong, two half-period current distributions on the slot edge. This phenomenon indicates that the antenna is operating in the $2^{\text {nd }}$-harmonic mode of slot at $f_{4}$. Comparing the simulation results (when $L_{l}=5.0 \mathrm{~mm}$ ) in Figs. 3 and 5, it is 
observed in the range: $2-6 \mathrm{GHz}$, that the parasitic patch has the following three contributions. First, it improves the impedance matching level for the first three modes, including the fundamental and $2^{\text {nd }}$-harmonic modes of the slot and the fundamental mode of the ground edge. Second, it has shifted the resonance frequency centers $\left(f_{1}, f_{2}\right.$, and $\left.f_{4}\right)$. For example, the first mode is red-shifted by $73 \mathrm{MHz}$, which aided the physical size reduction of the antenna. Third, it brings a new resonance mode (at $f_{3}$ ) due to its own physical structure, which fills in the gap of the already existing slot modes.
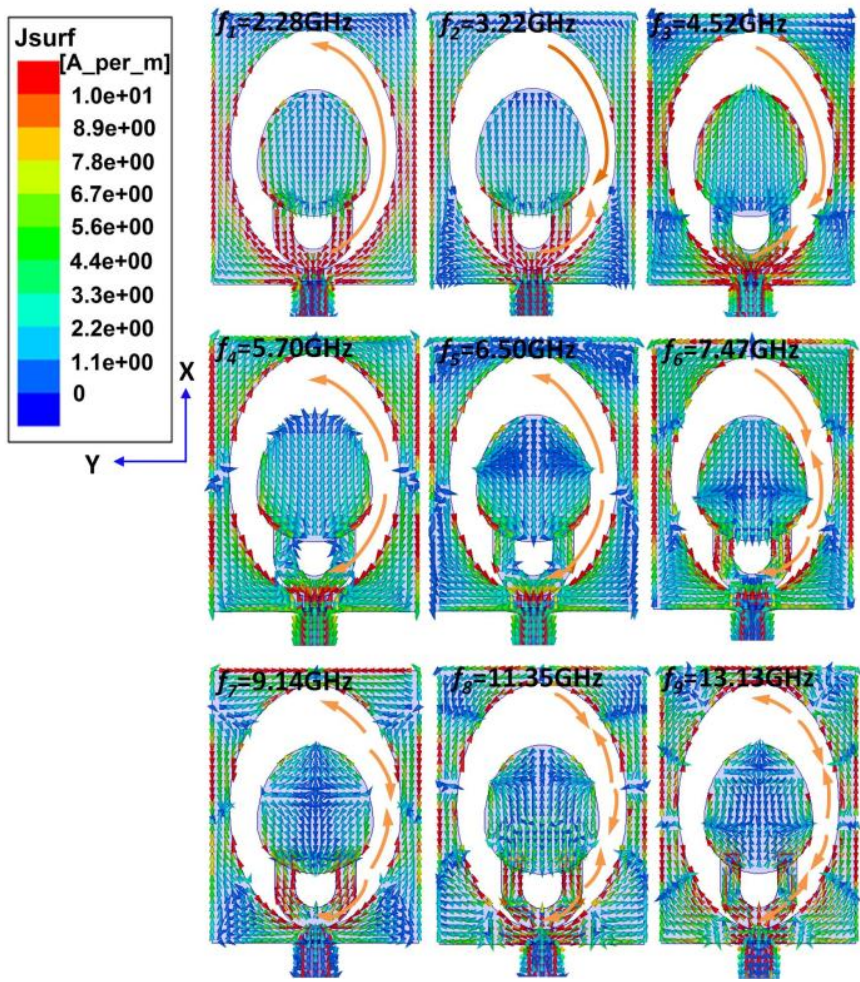

Fig. 8 Simulated current distributions on the ground, parasitic disc, and feeding structure of the antenna at the different frequencies labeled in Fig. 5.

The next several resonant frequency centers $\left(f_{5}-f_{9}\right)$ also have been inspected in a similar manner. It is seen in Fig. 8 that the $2^{\text {nd }}$-harmonic mode of the ground edge is located at $f_{5}$, which is close to $2 \times f_{2}=6.44 \mathrm{GHz}$. The $3^{\text {rd }}$-harmonic mode of the slot is located at $f_{6}$, which is close to $3 \times f_{l}=6.84 \mathrm{GHz}$. Since the length of the tuning stub that overlaps the slot is close to one quarter of the slot length (i.e., $d_{3}=12.873 \mathrm{~mm} \approx d_{1} / 4$ ), its fundamental mode resonance frequency is reasonably close to the $4^{\text {th }}$-harmonic mode of the slot. Therefore, there are three modes located around $f_{7}$ : the $4^{\text {th }}$-harmornic mode of the slot, the $2^{\text {nd }}$-harmonic mode of the parasitic patch, and the fundamental mode of the tuning stub. It can be observed that at $f_{7}$, the current on the stub is much stronger than in previous cases, which further confirms the existence of the fundamental mode of the tuning stub. Also, with the contribution of the other two harmonic modes, one now understand the presence of the two half-period and four half-period currents of nearly the same magnitudes on the parasitic patch and slot edges in Fig. 8. By comparing the $\left|S_{11}\right|$ values in Figs. 3 and 5, even though there are no explicit three dips which appear around $f_{7}$, the presence of the parasitic patch makes $\left|S_{11}\right|$ witness a $14.24 \mathrm{~dB}$ reduction in the
HFSS simulated values around $9.0 \mathrm{GHz}$. Moreover, since the antenna is operating simultaneously in three modes, its radiation pattern gets more directional and its gain is found to have a maximum: $6.81 \mathrm{~dB}$, as a consequence. Starting from $f_{8}$, the rest of the resonance frequencies originate mainly from the higher modes of the feed line with its tuning stub termination, the ground edge, the parasitic patch and the slot, and should appear in order in a sequence similar to that found for the resonance frequencies $f_{1}-f_{7}$. Consequently, by having two sets of resonances and by being able to adjust the dimensions of those elements to engineer the mutual couplings and, hence, the overlaps of these resonances, one is able to design the realized compact-sized hyper-band printed antenna.

\section{CONCLUSION}

A compact-sized elliptical-slot antenna augmented with a parasitic oval patch and fed with a microstrip line terminated in an elliptically-shaped tuning fork stub was proposed and studied numerically and experimentally. The measured results demonstrated that by optimizing the parasitic oval patch in the slot and the tuning fork stub, the antenna could exhibit a hyper-band -10dB-impedance bandwidth from 2.26 to 22.18 $\mathrm{GHz}$ and have reasonably stable radiation patterns, high radiation efficiencies and high gain values in a broad bandwidth. The measured performance characteristics are quite suitable for high fidelity, short pulse, and time domain applications. Moreover, because the hyper-band includes the standard UWB band, as well as the WLAN and WiMAX bands, it was emphasized that the antenna's performance characteristics were also suitable for single, multi-band, or UWB applications in this mobile platform portion of the hyper-band.

\section{APPENDIX STRUCTURAL PARAMETER ANALYSIS}

Since the parasitic patch plays an important role in achieving the hyper-band characteristics, its dimensions and position were parametrically investigated. The outcomes are presented in Figs. 9-11. In this parameter analysis, only one of the parameters was varied at a time, the others remaining unchanged. First, the effect of the overall size of the oval was studied; these $\left|S_{11}\right|$ results are given in Fig. 9. It was found that by changing the minor axis length of the oval $\left(b_{1}\right)$, the impedance matching in the lower bands was changed significantly, while it had little impact in the upper bands. When $b_{1}$ increases, the resonance frequency of fundamental mode of slot $\left(f_{l}\right)$ goes lower and the level of impedance matching increases. This occurs because when the patch size is increased, its capacitive coupling to the slot edge is also increased. Second, the results obtained by changing the value $r_{l}$ alone are given in Fig. 10. This change of the long axis length of one half of the oval again affects mainly the impedance matching level in the lower frequency range, and shifts the first several resonant frequencies to lower values. In fact, we were led to the oval shape because an elliptical one did not provide the necessary degree of freedom to tune the impedance matching level and shift each resonance frequency simultaneously. Finally, by changing the offset between the 
center of the slot and the center of the oval patch, the impedance matching characteristics across the entire hyper-band is affected, especially again in the lower band. This is demonstrated in Fig.11. When this offset is far enough away from the tuning fork stub, its mutual coupling ability becomes very weak. In contrast, when it is located quite close to the stub, it results in an over-coupling among the resonant elements, degrading the matching characteristics in the entire hyper-band. Modifications of the other design parameters had only minor impacts on the impedance matching; they were used only for fine tuning of the $\left|\mathrm{S}_{11}\right|$ values.

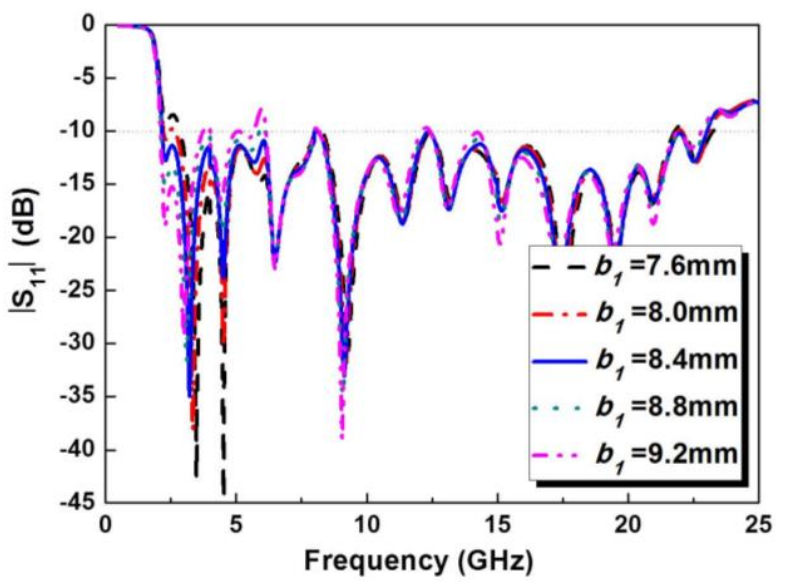

Fig. 9 Impact of the oval patch parameter $b_{1}$ (overall length) on the $\left|S_{11}\right|$ values of the antenna.

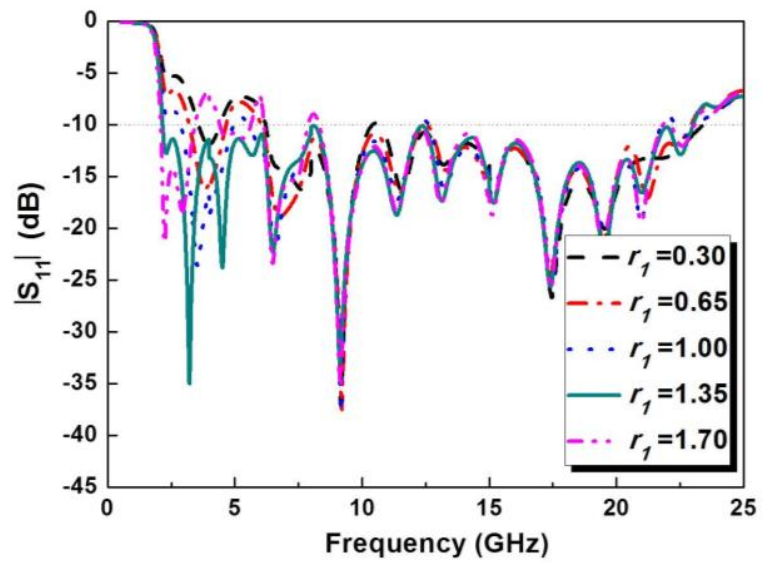

Fig. 10 Impact of the oval patch parameter $r_{1}$ on the $\left|S_{11}\right|$ values of the antenna.

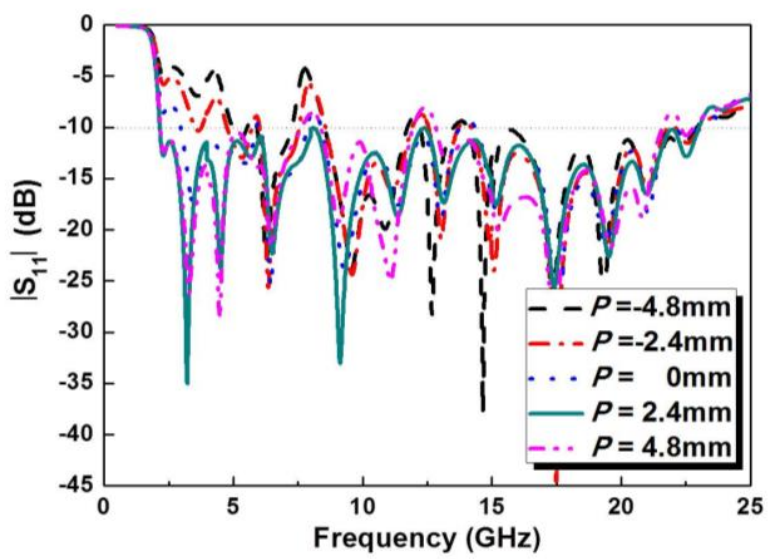

Fig. 11 Impact on the $\left|S_{11}\right|$ values of the offset, $p$, between the center of the oval patch and the center of the elliptical slot for the antenna.

\section{REFERENCES}

[1] M. Kahrizi, T. K. Sarkar, and Z. A. Maricevic, "Analysis of a wide radiating slot in the ground plane of a microstrip line," IEEE Trans. Microwave Theory Tech., vol. 41, no. 1, pp. 29-37, Jan. 1993.

[2] K. L. Wong, Compact and Broadband Microstrip Antennas. New York: Wiley, 2002.

[3] W. S. Chen, "A novel broadband design of a printed rectangular slot antenna for wireless communications," Microw. J., vol. 49, no. 1, pp. 122-130, Jul. 2006.

[4] P. Li, J. Liang, and X. Chen, "Study of printed elliptical/circular slot antennas for ultrawideband applications," IEEE Trans. Antennas Propag., vol. 54, no. 6, pp. 1670-1675, Jun. 2006.

[5] S.-W. Qu, C. Ruan, and B.-Z. Wang, "Bandwidth enhancement of wide-slot antenna fed by CPW and microstrip line," IEEE Antennas Wireless Propag. Lett., vol. 5, pp. 15-17, 2006.

[6] H. Eskandari, M. R. Booket, M. Kamyab, and M. Veysi, "Investigation on a class of wideband printed slot antenna," IEEE Antenna Wireless Propag. Lett., vol. 9, pp. 1221-1224, 2010.

[7] X. L. Liang, T. A. Denidni, L. N. Zhang, R. H. Jin, J. P. Geng, and Q. Yu, "Printed binomal-curved slot antennas for various wideband applications," IEEE Trans. Microw. Theory Tech., vol. 59, no. 4, pp. 1058-1065, Apr. 2011.

[8] O. A. Mashaal, S. K. A. Rahim, A. Y. Abdulrahman, M. I. Sabran, M. S. A. Rani, and P.S. Hall, "A coplanar waveguide fed two arm archimedean spiral slot antenna with improved bandwidth," IEEE Trans. Antennas Propag., vol. 61, no. 2, pp. 939-943, Feb. 2013.

[9] J.-Y. Jan, and L.-C. Wang, "Printed wideband rhombus slot antenna with a pair of parasitic strips for multiband applications," IEEE Trans. Antennas Propag., vol. 57, no. 4, pp. 1267-1270, Apr. 2009.

[10] S. Cheng, P. Hallbjörner, and A. Rydberg, "Printed slot planar inverted cone antenna for ultrawideband applications," IEEE Antennas Wireless Propag. Lett., vol. 7, pp. 18-21, 2008.

[11] T. Dissanayake and K. P. Esselle, "UWB performance of compact L-shaped wide slot antennas," IEEE Trans. Antennas Propag., vol. 56, no. 4, pp. 1183-1187, Apr. 2008.

[12] Y. Liu, K. L. Lau, Q. Xue, and C. H. Chan, "Experimental studies of printed wide-slot antenna for wide-band applications," IEEE Antennas Wireless Propag. Lett., vol. 3, pp. 273-275, Dec. 2004.

[13] W.-S. Chen and F.-M. Hsieh, "A broadband design for a printed isosceles triangular slot antenna for wireless communications," Microw. J., vol. 48, no. 7, pp. 98-112, Jul. 2007.

[14] S. -W. Qu, J. -L Li, J. -X. Chen, and Q. Xue, "Ultrawideband strip-loaded circular slot antenna with improved radiation patterns," IEEE Trans. Antennas Propag.,vol.55,no.11,pp. 3348-3353, Nov. 2007.

[15] T. A. Denidni and M.A. Habib, "Broadband printed CPW-fed circular slot antenna," Electron. Lett., vol. 42, no. 3, pp. 135 - 136 Feb. 2006.

[16] W. -L. Chen, G. -M. Wang, and C.-X. Zhang, "Bandwidth enhancement of a microstrip-line-fed printed wide-slot antenna with a fractal-shaped slot," IEEE Trans. Antennas Propag., vol. 57, no. 7, pp. 2176-2179, Jul. 2009.

[17] D. D. Krishna, M. Gopikrishna, C. K. Anandan, P. Mohanan, and K. Vasudevan, "CPW-fed Koch fractal slot antenna for WLAN/WiMAX applications," IEEE Antennas Wireless Propag. Lett., vol. 7, pp. 389-392, 2008.

[18] D. D. Krishna M. Gopikrishna C.K. Aanandan P. Mohanan, and K. Vasudevan, "Compact wideband Koch fractal printed slot antenna," IET Microw. Antennas Propag., vol. 3, no. 5, pp. 782-789, 2009.

[19] J.-Y. Jan and J.-W. Su, "Bandwidth enhancement of a printed wide-slot antenna with a rotated slot," IEEE Trans. Antennas Propag., vol. 53, no. 6, pp. 2111-2114, Jun. 2005.

[20] Y. Sung, "Bandwidth enhancement of a microstrip line-fed printed wide-slot antenna with a parasitic center patch," IEEE Trans. Antennas Propag., vol. 60, no. 4, pp. 1712-1716, Apr. 2012.

[21] S. T. Fan, Y. Z. Yin, B. Lee, W. Hu, and X. Yang, "Bandwidth enhancement of a printed slot antenna with a pair of parasitic patches," IEEE Antennas Wireless Propag. Lett., vol. 11, pp. 1230-1233, 2012.

[22] D. V. Giri and F. M. Tesche, "Classification of intentional 
electromagnetic environments (IEME)," IEEE Trans. Electromag. Compat., vol. 46, pp. 322 -328, Aug. 2004.

[23] J. Y. Sze, and K. L. Wong, "Bandwidth enhancement of a microstrip line-fed printed wide-slot antenna," IEEE Trans. Antennas Propag., vol. 49, no. 7, pp. 1020-1024, Jul. 2001.

[24] M.-C. Tang, and R. W. Ziolkowski, "Compact, two-element array with high broadside directivity," IET Microw. Antennas Propag., vol.7, no. 8, pp. 663-671, Jun. 2013.

[25] H. Tehrani, and K. Chang, "Multifrequency operation of microstrip-fed slot-ring antennas on thin low-dielectric permittivity substrates," IEEE Trans. Antennas Propag., vol. 50, no. 9, pp. 1299-1308, Sep. 2002.

[26] M.-H. Ho and G.-L. Chen, "Reconfigured slot-ring antenna for 2.4/5.2 GHz dual-band WLAN operations," IET Microw. Antennas Propag., vol. 1, no. 3, pp. 712-717, Jun. 2007.

[27] J. Lacik, and T. Mikulasek "Circular ring-slot antenna fed by SIW for WBAN applications," Proc. 7th European Conference on Antennas and Propagation (EuCAP), Gothenburg, Sweden, pp. 213-216, Apr. 2013.

[28] J. Liang, L. Guo, C.C. Chiau, X. Chen and C.G. Parini, "Study of CPW-fed circular disc monopole antenna for ultra wideband applications," IEE Proc. Microw. Antennas Propag., vol. 152, no. 6, pp. 520-526, Dec. 2005.

[29] H. Zhang, R. Zhou, Z. Wu, H. Xin, and R. W. Ziolkowski, "Designs of ultra wideband (UWB) printed elliptical monopole antenna with slots," Microw. Opt. Technol. Lett., vol. 52, No. 2, pp. 466-471, Feb. 2010.

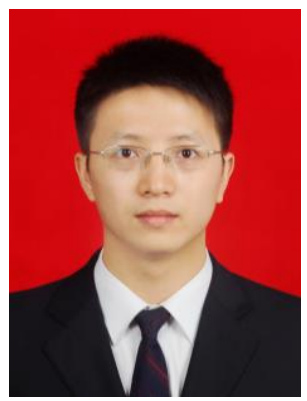

Ming-Chun Tang (S'12-M'13) received the B. S degree in physics from the Neijiang Normal University, Neijiang, China, in 2005 and the Ph. D. degree in radio physics from the University of Electronic Science and Technology of China (UESTC), in 2013.

From August 2011 to August 2012, he was also with the Department of Electrical and Computer Engineering, The University of Arizona, Tucson, AZ, USA, as a Visiting Scholar. He is currently an Assistant Professor in the College of Communication Engineering, Chongqing University, China. His research interests include electrically small antennas, RF circuits, metamaterial designs and their applications.

Prof. Tang was a recipient of the Best Student Paper Award in the 2010 International Symposium on Signals, Systems and Electronics (ISSSE2010) held in Nanjing, China. He is serving as a reviewer for IEEE/IET journals including the IEEE Antennas and Wireless Propagation Letters, IEEE Antennas and Propagation Magazine, and IET Microwaves, Antennas \& Propagation.

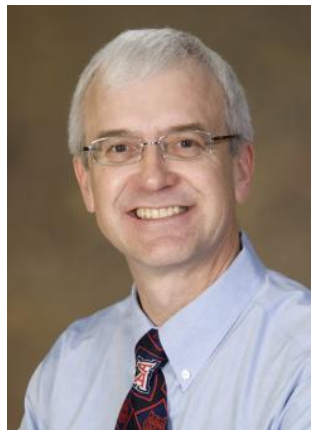

Richard W. Ziolkowski (M'97-SM'91-F'94) received his Sc.B. degree in physics, magna cum laude with honors, from Brown University (1974), and his M.S. (1975) and Ph.D. (1980) degrees in physics from the University of Illinois at Urbana-Champaign. He is the Litton Industries John M. Leonis Distinguished Professor in the Department of Electrical and Computer Engineering at the University of Arizona. He is also a Professor in the College of Optical Sciences at the University of Arizona. He was awarded an Honorary Doctorate, Doctor Technish Honoris Causa, from the Technical University of Denmark (DTU) in 2012. He was the Computational Electronics and Electromagnetics Thrust Area Leader in the Engineering Research Division at the Lawrence Livermore National Laboratory before joining the University of Arizona in 1990.

Prof. Ziolkowski is a Fellow of both the Institute of Electrical and Electronics Engineers (IEEE) and the Optical Society of America (OSA). He served as the President of the IEEE Antennas and Propagation Society in 2005. $\mathrm{He}$ is also actively involved with the URSI, OSA and SPIE professional societies. He and Prof. Nader Engheta, University of Pennsylvania, are Co-Editors of the best-selling 2006 IEEE-Wiley book, Metamaterials: Physics and Engineering Explorations.

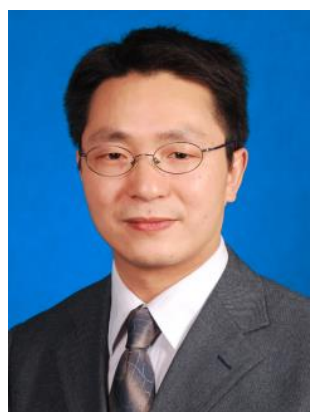

Shaoqiu Xiao (M'05) received Ph.D. degree in Electromagnetic field and Microwave Engineering from the University of Electronic Science and Technology of China (UESTC), Chengdu, China, in 2003. From January 2004 to June 2004, he joined UESTC as an assistant professor. From July 2004 to March 2006, he worked for the Wireless Communications Laboratory, National Institute of Information and Communications Technology of Japan (NICT), Singapore, as a researcher with the focus on the planar antenna and smart antenna design and optimization. From July 2006 to June 2010, he worked for UESTC as an associate professor and now he is working for UESTC as a professor. His current research interests include planar antenna and phased array, microwave passive circuits and time reversal electromagnetics. 